\title{
Birth-death process of local structures in defect turbulence described by the one-dimensional complex Ginzburg-Landau equation
}

\author{
Yusuke Uchiyama, Hidetoshi Konno \\ Department of Risk Engineering, Faculty of Information and Systems, University of Tsukuba, Tsukuba, Ibaraki 305-8573, Japan
}

\begin{abstract}
Defect turbulence described by the one-dimensional complex Ginzburg-Landau equation is investigated and analyzed via a birthdeath process of the local structures composed of defects, holes, and modulated amplitude waves (MAWs). All the number statistics of each local structure, in its stationary state, are subjected to Poisson statistics. In addition, the probability density functions of interarrival times of defects, lifetimes of holes, and MAWs show the existence of long-memory and some characteristic time scales caused by zigzag motions of oscillating traveling holes. The corresponding stochastic process for these observations are fully described by a non-Markovian master equation.
\end{abstract}

Keywords: Complex Ginzburg-Landau equation, Defect turbulence, Local structures, Poisson statistics, Long-memory, Non-Markovian master equation

\section{Introduction}

The complex Ginzburg-Landau equation (CGLE) is the simplest model describing spatiotemporal patterns in nonequilibrium open systems [1], especially near Hopf bifurcations [2]. Defect dynamics is regarded as a key physical concept in understanding the nature of complex systems such as topological defects in thermal fluid convection [3], catalytic CO oxidation on Pt surfaces [4], and cardiac sudden death [5]. Recently, the Bekki-Nozaki (BN) hole, a defect of the one-dimensional (1D) CGLE, has been discovered in a healthy human heart, suggesting that nonlinear waves play an important role in understanding the nature of both regular and irregular human heart behavior [6].

The scaled CGLE is expressed by a complex order parameter $A$ with two real parameters $\left(c_{1}, c_{2}\right)$ :

$$
\partial_{t} A=A+\left(1+i c_{1}\right) \nabla^{2} A-\left(1+i c_{2}\right)|A|^{2} A .
$$

Spatiotemporal patterns described by the CGLE are specified by the sets of $\left(c_{1}, c_{2}\right)$ with a system size corresponding to the appearance of various instabilities and transitions $[7,8]$.

A class of traveling wave solutions, $A(x, t)=\hat{A}(x-v t) \mathrm{e}^{-i \omega t}$, also known as coherent structures, has been introduced to classify local structures of the 1D CGLE [9]. Substituting the ansatz into Eq. (1), one obtains a set of coupled first order ordinary differential equations (ODEs) with two real parameters $(v, \omega)$ :

$$
\begin{aligned}
& \partial_{\xi} a=\kappa a, \\
& \partial_{\xi} z=-z^{2}+\frac{1}{1+i c_{1}}\left[-1-i \omega+\left(1+i c_{2}\right) a^{2}-v z\right],
\end{aligned}
$$

Email address: r1230160@risk.tsukuba.ac.jp (Yusuke Uchiyama) where $\xi=x-v t, a=|\hat{A}|$, and $z$ is defined by $z \equiv \partial_{\xi} \ln (A)=$ $\kappa+i q$. The local structures display different trajectories in threedimensional (3D) phase space: A plane wave corresponds to a stable fixed point [9]; a homoclinic hole is defined as an unstable homoclinic orbit associated with a saddle node bifurcation [10, 11], whereas a modulated amplitude wave (MAW) is defined as the stable one $[12,13]$; and the $\mathrm{BN}$ hole corresponds to an unstable heteroclinic orbit $[9,10]$.

The concept of coherent structures has been extended to spatiotemporal disorder of the 1D CGLE. The spatiotemporal intermittency is modeled by a coupled map lattice involved in defects and holes, which reflect the features of the $\mathrm{BN}$ holes and the homoclinic holes, in the disorder background [11]. The phase turbulence is regarded as a state of many-body interactions among the MAWs with their associated instabilities [13].

Although the above approaches based on local structures have been able to capture various dynamical properties of systems with disorder, their statistical properties have not been fully investigated. In this research, we show that the defect turbulence can be described by a birth-death process composed of defects, holes, and MAWs based on a non-Markovian master equation.

\section{Identification of the local structures}

We have implemented a numerical simulation of Eq. (1) by using a fourth-order Runge-Kutta scheme for time and a second-order central-difference scheme for space with periodic boundary condition. The time and space resolutions are $\Delta t=0.01$ and $\Delta x=0.5$, respectively. The parameters of Eq. (1) are fixed at $\left(c_{1}, c_{2}\right)=(1.5,-1.2)$, beyond the Benjamin-Feir (BF) criterion with system size $\Omega=500$ large enough to generate defect turbulence [7]. Figure 1 shows the spatiotemporal 

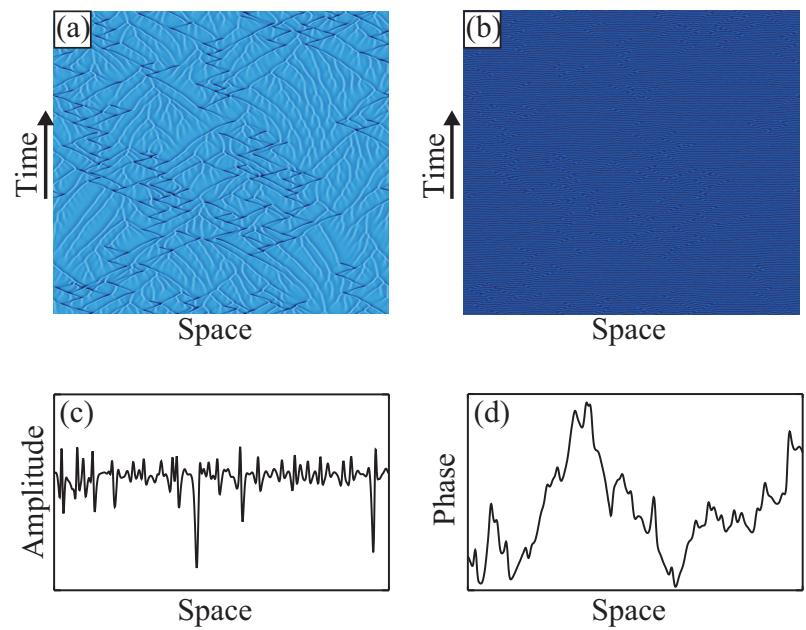

Figure 1: Spatiotemporal patterns of (a) the amplitude $|A|$ and (b) the phase $\arg (A)$ with snapshots of (c) the amplitude and (d) the phase at a certain time. Black and white lines in (a) correspond to the local minima and maxima of the amplitude in (c), respectively. The discontinuities of color gradation in (b) and the phase jumps in (d) are accompanied by the local minima of the amplitude.

pattern of (a) the amplitude $|A|$ and (b) the phase $\arg (A)$ with snapshots of (c) the amplitude and (d) the phase at a certain time. Black and white lines in Fig. 1(a) correspond to local minima and maxima in Fig. 1(c), respectively. The black lines accompany discontinuities, namely, phase jumps in Fig. 1(b). The range of phase values, as seen in Fig. 1(d), is extended to get unbounded lines for evaluating phase gradients adequately. We utilize information about the local profiles of both amplitude and phase to identify the types of local structures appropriately. In what follows, let us review their definitions in the defect turbulence [14].

A defect is characterized by a nonzero winding number, and thus we can capture defects as phase singular points in the $x-t$ plane by the criterion $\frac{1}{2 \pi} \oint \nabla \varphi \cdot \mathrm{d} \mathbf{r} \neq 0$, where $\varphi=\arg (A)$. The contour integrals have been numerically evaluated on each discrete lattice $(i, j)$, being discretized as $i=x / \Delta x$ for space and $j=t / \Delta t$ for time, in terms of the phase on site, $\varphi_{i, j}$. A defect is captured if and only if its phase discontinuity vanishes on the integral contour: $\varphi_{i-1, j-1} \rightarrow \varphi_{i+1, j-1} \rightarrow \varphi_{i+1, j+1} \rightarrow \varphi_{i-1, j+1} \rightarrow \varphi_{i-1, j-1}$ (see Fig. 2(a)). Lifetimes of defects are thereby less than the discretized time interval $\Delta t$.

A hole is defined as a local minimum of amplitude with a phase jump (see Fig. 2(b)). Although defects, for which the winding numbers are not zero, also satisfy this condition, the winding numbers of holes are zero. In other words, phase jumps of holes pass through their integral contours without vanishing. Hence we can distinguish holes from defects.

As mentioned above, a MAW appears as the stable branch of a saddle node bifurcation whereas a hole appears as the unstable one in the context of coherent structures. It is thus difficult to distinguish MAWs from holes in the defect turbulence, where both the stable and the unstable branches coexist. A MAW is defined by a local maximum of amplitude with a steep phase gradient exceeding a specific value (see Fig. 2(c)). To avoid

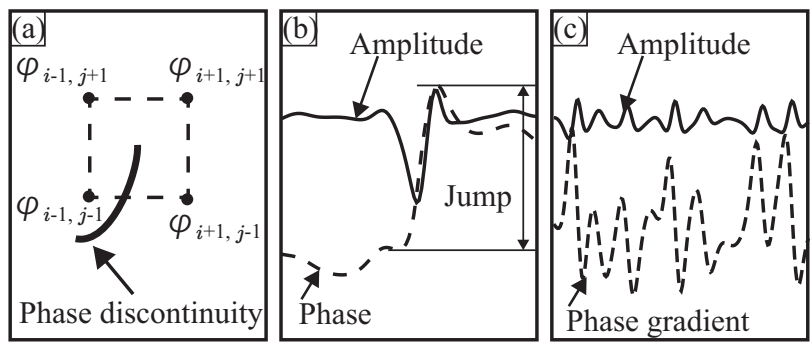

Figure 2: Conceptual diagram of the definition of the local structures. (a) Defects are identified by phase singularities at which the winding numbers in the discretized $x-t$ plane are not zero. A defect is thus captured if and only if a phase discontinuity vanishes on the discretized integral contour: $\varphi_{i-1, j-1} \rightarrow \varphi_{i+1, j-1} \rightarrow \varphi_{i+1, j+1} \rightarrow \varphi_{i-1, j+1} \rightarrow \varphi_{i-1, j-1}$. (b) Holes are identified by local minima of the amplitude at phase jumps. Holes can thereby be captured after defect identification because defects also have the same characteristic. (c) MAWs are identified by local maxima of the amplitude with steep phase gradients exceeding a specific value.

confusing MAWs with holes in the defect turbulence, we have performed the procedure for MAWs after defects and holes had been identified completely.

Note that defects need "three" snapshots of successive time steps to be captured by evaluating the contour integrals, whereas holes and MAWs are captured in "one" snapshot of each time step. However, many previous authors $[15,16]$, including us $[17,18]$, have used one snapshot of each time step to identify local structures, which gives a misleading identification that all the local depressions of amplitude are regarded as the BN-holes. Furthermore, defect dynamics in 1D systems are essentially different from those in two-dimensional (2D) systems. The latter defects are captured in "one" snapshot of each time step, where the contour integrals can be evaluated in $x-y$ plane.

\section{Statistical analysis of the local structures}

\subsection{Number statistics of defects, holes, and MAWs}

After identifying the local structures, we investigated their statistics. Figure 3 shows the probability density functions (PDFs) of the number of (a) defects, (b) holes, and (c) MAWs. The number of each local structure is counted in the whole space at each time step of the numerical simulation. The Fano factor $(F F)$ is defined as the variance-to-man ratio, of which the Poisson distribution is 1 . The specific values of the $F F$ are (a) 1.00 for defects, (b) 1.01 for holes, and (c) 1.04 for MAWs. Thus, all these PDFs are subjected to the Poisson distribution

$$
P(n)=\frac{\lambda^{n}}{n !} \mathrm{e}^{-\lambda},
$$

where $n$ is the number of each local structure and $\lambda$ is the constant parameter estimated as the average value of $n$.

In our previous works $[17,18]$, we reported that the number of local minima in amplitude, regarded as "holes" in that work, are subjected to sub-Poisson statistics, which implies the existence of bunching states analogous to the squeezed states 

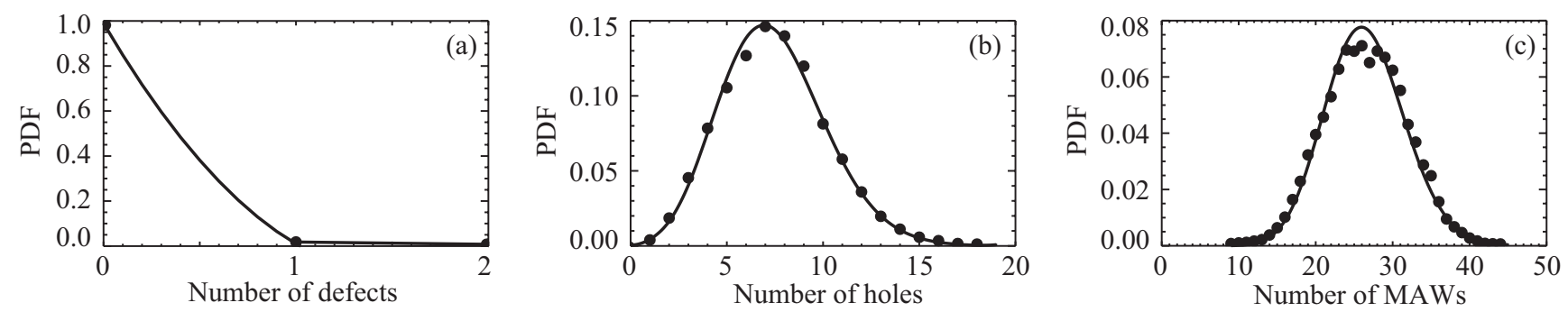

Figure 3: PDFs of the number of (a) defects, (b) holes, and (c) MAWs. The number of each local structure is counted in the whole space at each time step of the numerical simulation. The black circles indicate the collected data from the numerical simulation and the solid lines represent the Poisson distributions with estimated parameters of (a) $\lambda=0.0105$, (b) $\lambda=7.43$, and (c) $\lambda=26.5$.
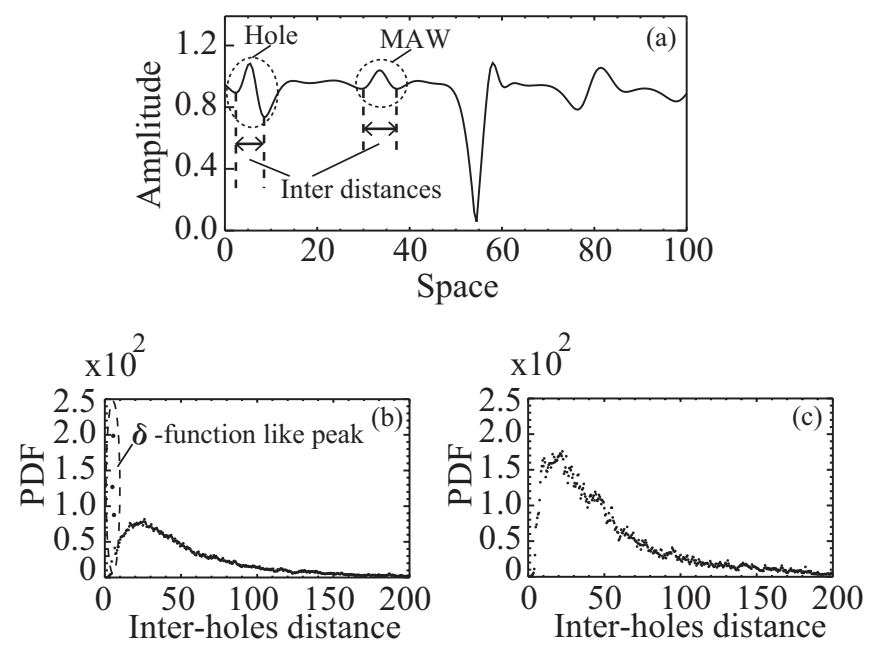

Figure 4: (a) Distances between nearby amplitude dips, regarded as the interhole distances in our previous works [17, 18]. However, the amplitude dips are in fact parts of either holes or MAWs. The PDFs are shown for interhole distances obtained from (b) the method with only an amplitude value or (c) the method presented in the previous section. The $\delta$-function-like peak in (b) is automatically removed from (c), which means that the identification method based on only the amplitude causes confusion with regard to the sub-Poisson statistics.

of light (photon bunching). Actually, one can recognize a $\delta$ function-like peak at a certain low value in the PDF of the interhole distances, which is caused by nearby amplitude dips of holes and MAWs (see Figs. 4(a) and (b)). This $\delta$-functionlike peak is automatically removed by the hole identification method presented in the previous section (see Fig. 4(c)). Thus the sub-Poisson statistics for the number of the local structures can be regarded as an unexpected illusion caused by an unsuitable identification method based on only amplitude value.

The interaction rules of the local structures have been investigated to understand the dynamical properties of systems with disorder. A hole can transform into a defect when the amplitude dip of the hole reaches zero, followed by the defect generating another hole and transforming back to a hole [20]. Holes and MAWs transform into each other through saddle-node bifurcations [14]. MAWs emerge from and sink into the background; moreover, splitting and merging processes of MAWs are dominant in turbulent regimes [13]. The observed main interaction rules can be written as

$$
\begin{aligned}
& H \underset{k_{2}}{\stackrel{k_{1}}{\rightleftarrows}} D, \\
& D \stackrel{k_{3}}{\rightarrow} D+H, \\
& H \underset{k_{5}}{\stackrel{k_{4}}{\rightleftarrows}} M, \\
& 0 \underset{k_{7}}{\stackrel{k_{6}}{\rightleftarrows}} M, \\
& M \underset{k_{9}}{\stackrel{k_{8}}{\rightleftarrows}} 2 M,
\end{aligned}
$$

where $D, H, M$, and $k_{i}$ denote a defect, a hole, a MAW, and their reaction rates, respectively. The corresponding Markovian master equation derived from the above interaction rules is

$$
\begin{aligned}
\frac{\mathrm{d}}{\mathrm{d} t} P(d, h, m ; t) & =k_{1}(d+1) P(d+1, h-1, m ; t) \\
& +k_{2}(h+1) P(d-1, h+1, m ; t) \\
& +k_{3} d P(d, h-1, m ; t) \\
& +k_{4}(h+1) P(d, h+1, m-1 ; t) \\
& +k_{5}(m+1) P(d, h-1, m+1 ; t) \\
& +k_{6} \Omega P(d, h, m-1 ; t) \\
& +k_{7}(m+1) P(d, h, m+1 ; t) \\
& +k_{8}(m-1) P(d, h, m-1 ; t) \\
& +k_{9} \Omega^{-1}(m+1) m P(d, h, m+1 ; t) \\
& -\left[k_{1} d+k_{2} h+k_{3} d+k_{4} h+k_{5} m+k_{6} \Omega\right. \\
& \left.+k_{7} m+k_{8} m+k_{9} \Omega^{-1} m(m-1)\right] \\
& \times P(d, h, m ; t),
\end{aligned}
$$

where $d, h$, and $m$ denote the number of defects, holes, and MAWs, and $\Omega$ is the system size. In general, a multivariable Poisson distribution is derived from the master equations for stationary states under the independence condition [21]. Thus, assuming that $P(d, h, m ; t)=P_{0}(d, h, m ; t)+\epsilon P_{1}(d, h, m ; t)$ with $P_{0}(d, h, m ; t)=\xi(d ; t) \eta(h ; t) \zeta(m ; t)$ and $\epsilon$ decaying more rapidly than $\Omega^{-1}$ with $\Omega \rightarrow \infty$, we can obtain the evolution equations of 
the marginal PDFs, $\xi(d ; t), \eta(h ; t)$, and $\zeta(m ; t)$ from Eq. (5) as

$$
\begin{aligned}
\frac{\mathrm{d}}{\mathrm{d} t} \xi(d ; t) & =k_{1}(d+1) \xi(d+1 ; t)+k_{2}\langle h\rangle_{\eta} \xi(d-1 ; t) \\
& -\left[k_{1} d+k_{2}\langle h\rangle_{\eta}\right] \xi(d ; t), \\
\frac{\mathrm{d}}{\mathrm{d} t} \eta(h ; t) & =\left(k_{2}+k_{4}\right)(h+1) \eta(h+1 ; t) \\
& +\left(k_{1}+k_{3}\right)\langle d\rangle_{\xi} \eta(h-1 ; t) \\
& -\left[\left(k_{2}+k_{4}\right) h+\left(k_{1}+k_{3}\right)\langle d\rangle_{\xi}\right] \eta(h ; t), \\
\frac{\mathrm{d}}{\mathrm{d} t} \zeta(m ; t) & =\left(k_{9} \Omega^{-1} m+k_{5}+k_{7}\right)(m+1) \zeta(m+1 ; t) \\
& +\left[k_{4}\langle h\rangle_{\eta}+k_{8}(m-1)+k_{6} \Omega\right] \zeta(m-1 ; t) \\
& -\left[k_{9} \Omega^{-1}(m-1)+k_{5}+k_{7}\right] m \zeta(m ; t) \\
& -\left[k_{4}\langle h\rangle_{\eta}+k_{8} m+k_{6} \Omega\right] \zeta(m ; t),
\end{aligned}
$$

where $\langle d\rangle_{\xi}$ and $\langle h\rangle_{\eta}$ are the mean values of $\xi$ and $\eta$ with respect to $\xi(d ; t)$ and $\eta(h ; t)$, respectively. Because the PDFs of the number of each local structure are evaluated for the stationary state, the stationary PDFs, $\xi_{s}(d), \eta_{s}(h)$, and $\zeta_{s}(m)$, in a large system where $\epsilon \rightarrow 0$, are readily obtained from Eq. (6) as the Poisson distributions:

$$
\begin{aligned}
& \xi_{s}(d)=\frac{\lambda_{\xi}^{d}}{d !} \mathrm{e}^{-\lambda_{\xi}}, \quad \lambda_{\xi}=\frac{k_{2}\langle h\rangle_{\eta}}{k_{1}}, \\
& \eta_{s}(h)=\frac{\lambda_{\eta}^{h}}{h !} \mathrm{e}^{-\lambda_{\eta}}, \quad \lambda_{\eta}=\frac{\left(k_{1}+k_{3}\right)\langle d\rangle_{\xi}}{k_{2}+k_{4}} \\
& \zeta_{s}(m)=\frac{\lambda_{\zeta}^{m}}{m !} \mathrm{e}^{-\lambda_{\zeta}}, \quad \lambda_{\zeta}=\frac{k_{6} \Omega}{k_{5}+k_{7}} .
\end{aligned}
$$

The Markovian master equations can be written in the form

$$
\frac{\mathrm{d}}{\mathrm{d} t} P(n ; t)=J(n+1 ; t)-J(n ; t),
$$

where $J(n ; t)$ is a functional of $P(n ; t)$, the stochastic flux [21]. In the stationary state, $J_{s}(n)=0$ leads to the recursion relation

$$
P(n)=\frac{c(n-1)}{a(n)} P(n-1)
$$

with $n$-dependent creation rate $c(n)$ and annihilation rate $a(n)$. The ratio, $c(n-1) / a(n)$ must be proportional to $n^{-1}$ for stationary Poisson processes. Figure 5 shows the creation and the annihilation rates of the local structures estimated by using the least-square method. The creation rates (Fig. 5(a), (c), and (e)) are estimated as constant values, $c(n)=c_{0}$, and the annihilation rates (Fig. 5(b), (d), and (f)) are proportional to $n, a(n)=a_{1} n$. The values of the creation and the annihilation rates for smaller numbers of MAWs less than 21 were also counted. In estimating the creation and the annihilation rates of MAWs, we have utilized the data for the values of them for the numbers for $21 \leq$ $n \leq 45$, since the error bar increases for smaller values of $n$ in $c_{0}$, and for larger values of $n$ in $a_{1}$. Hence, the values $c_{0}, a_{1}$ for $21 \leq n \leq 45$ are depicted in Figs. 5(e), (f). This result gives a plausible explanation for the appearance of Poisson statistics for the stationary state. (a)

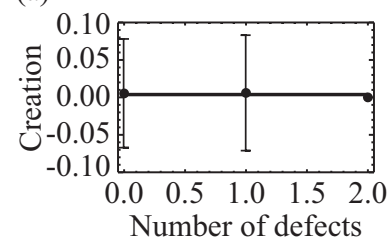

(b)

(c)

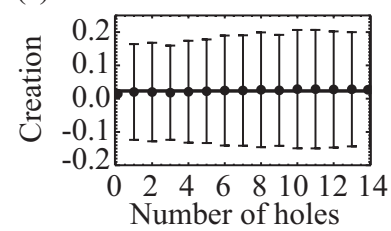

(e)

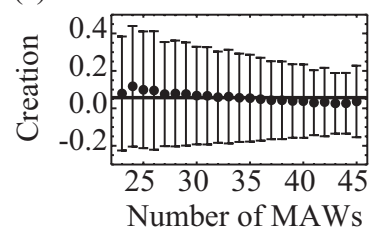

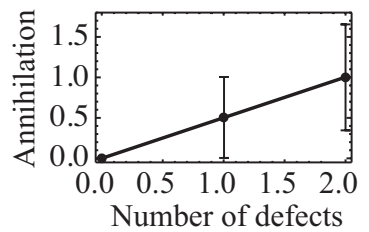

(d)
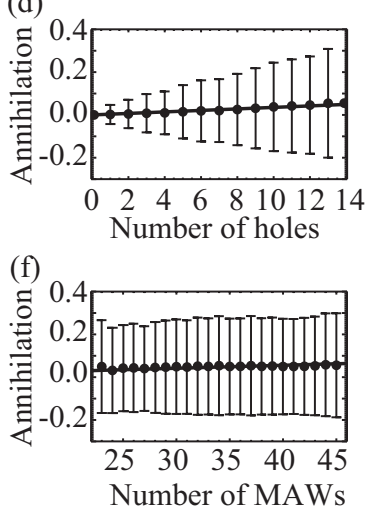

Figure 5: Creation rates of (a) defects, (c) holes, and (e) MAWs, and annihilation rates of (b) defects, (d) holes, and (f) MAWs. These creation and annihilation rates are estimated by using the least-squares method. In each figure, the black circles indicate the mean values with the vertical bars indicating the standard deviations obtained from the numerical simulation. The values of each creation and annihilation rate, $\left(c_{0}, a_{1}\right)$, are $(0.00376,0.501)$ for defects, $(0.0232,0.0037)$ for holes, and $(0.057,0.00147)$ for MAWs.

\subsection{Long-memory and characteristic time scales in interar- rival times of defects and lifetimes of holes and MAWs}

The statistical properties of interarrival times of defects and lifetimes of holes and MAWs give us information on the time code for the birth-death process of the local structures, while the number statistics provide information on the rate code. These two codes provide complementary information. To obtain the PDFs of the interarrival times of defects and the lifetimes of holes and MAWs, we have to know the time evolution of the PDFs of the number of the local structures. The Markovian master equation of each local structure in the large system is

$\frac{\mathrm{d}}{\mathrm{d} t} P(n ; t)=c_{0}[P(n-1 ; t)-P(n ; t)]+a_{1}[(n+1) P(n+1 ; t)-n P(n ; t)]$.

The exact solution is given by

$$
P(n ; t)=\frac{1}{n !}\left[\frac{c_{0}}{a_{1}}\left(1-\mathrm{e}^{-a_{1} t}\right)\right]^{n} \exp \left[-\frac{c_{0}}{a_{1}}\left(1-\mathrm{e}^{-a_{1} t}\right)\right] .
$$

The corresponding PDFs of the interarrival times of defects and the lifetimes of holes and MAWs, $f_{M}(t)$, are readily obtained from the relation $f_{M}(t)=-\frac{\mathrm{d}}{\mathrm{d} t} P(0 ; t)$ as

$$
f_{M}(t)=c_{0} \mathrm{e}^{-a_{1} t} \exp \left[-\frac{c_{0}}{a_{1}}\left(1-\mathrm{e}^{-a_{1} t}\right)\right] .
$$

Figure 6 shows the PDFs obtained from the numerical simulation; these PDFs are characterized by fat tails and specific 

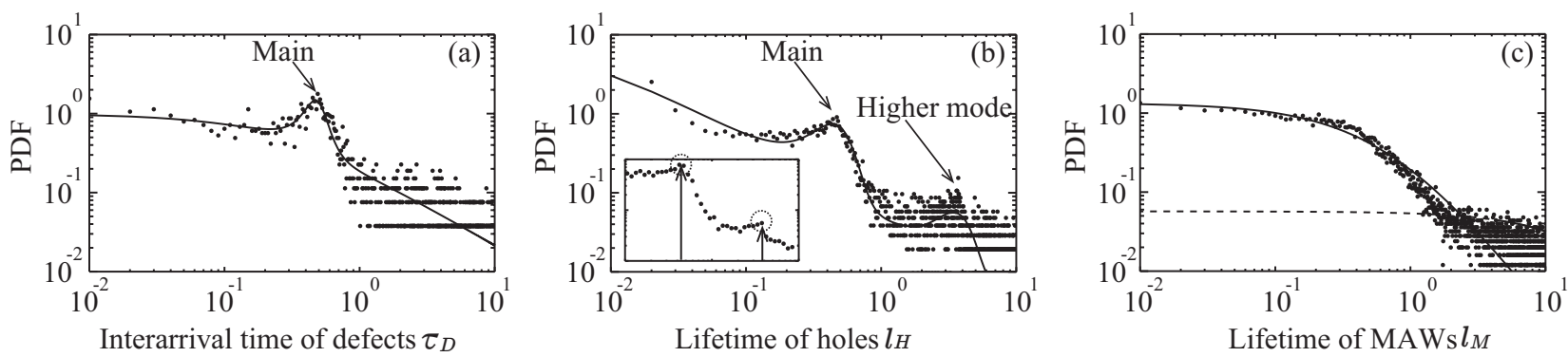

Figure 6: PDFs of (a) the interarrival times of defects $\tau_{D}$ and the lifetimes (b) of holes $l_{H}$ and (c) of MAWs $l_{M}$. The window in (b), where one can see the main and higher mode peaks, is a enlarged view of averaged data. In each figure, the black circles represent the numerically obtained PDF and the solid line is the theoretical PDF of the non-Markovian model $f_{n M}(\cdot)$. The dashed line in (c) is the theoretical PDF of the Markovian model $f_{M}\left(l_{M}\right)$. The parameters of the theoretical PDFs are estimated by using the least-square method as follows: (a) $\left(c_{0}, a_{1}, \kappa_{00}, \kappa_{10}, T_{0}, \alpha_{0}, v_{0}, v_{1}\right)=\left(3.76 \times 10^{-3}, 0.501,3.72,59.5,0.48,1.53,3.7 \times 10^{-4}, 4.23\right),(\mathrm{b})$ $\left(c_{0}, a_{1}, \kappa_{00}, \kappa_{10}, T_{0}, \alpha_{0}, \kappa_{01}, \kappa_{11}, T_{1}, \alpha_{1}, v_{0}, v_{1}\right)=\left(2.32 \times 10^{-2}, 3.7 \times 10^{-3}, 7.0,6.65,0.48,4.43,3.74,7.16 \times 10^{-2}, 3.76,7.65,3.02 \times 10^{-2}, 164.0\right)$, and $(\mathrm{c})\left(c_{0}, a_{1}, v_{0}, v_{1}\right)=$ $\left(5.7 \times 10^{-2}, 1.47 \times 10^{-3}, 30.1,1.28\right)$

peaks. In contrast, the theoretical PDF, $f_{M}(t)$, in Eq. (12) shows a double-exponential decay.

Recalling the spatiotemporal dynamics of the defect turbulence in Fig. 1, one can see zigzag motions of traveling holes with oscillating amplitudes. Figures 7(a) and (b) show a zigzag motion of a traveling hole extracted from Fig. 1 and a defect generation caused by the traveling hole moving from the right side to the left side, respectively. In Fig. 7(a), the white circles on the edges of the zigzag black line correspond to defects generated from the traveling hole. In Fig. 7(b), the hole moving from the right side changes to a defect, which generates another hole moving to the left side when the amplitude of the traveling hole reaches zero. The defect then changes into a hole moving to the right side. Hence the intervals between nearest neighbor defects in the direction of time in Fig. 7(a) correspond to both the interarrival times of defects and the lifetimes of holes. In other words, a kind of periodicity involved in the zigzag motions of holes causes the specific peaks in Figs. 6(a) and (b).

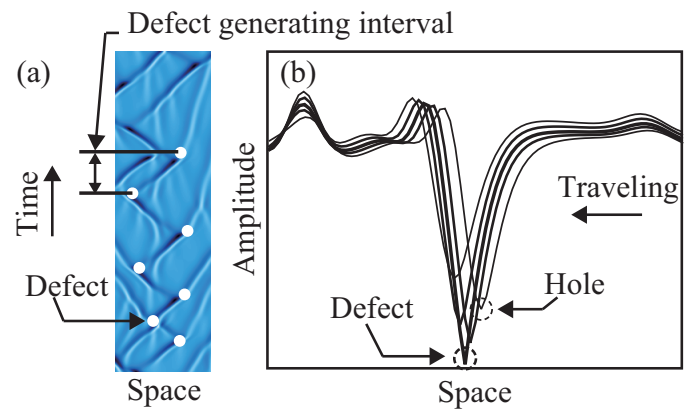

Figure 7: (a) Zigzag motion of a traveling hole extracted from Fig. 1(a). The black line is a trajectory of the traveling hole and the white circles are defects. The intervals between nearest neighbor defects in the direction of time correspond to both the interarrival times of defects and the lifetimes of the holes. (b) Defect generation caused by the traveling hole moving from the right side to the left side. The defect is generated when the amplitude of the traveling hole reaches zero.

We assume that the zigzag motions of holes enhance the creation rates of defects and holes as a superposition of Lorentziantype functions involved in periods of the zigzag motions. In addition, a time-scaling function is introduced to realize the effects of long-memory. There are two ways to take into account the effect of long-memory for master equations: 1. generalized master equations with convolution-type memory functions involved in waiting-time PDFs of reactions [22] and 2. nonstationary, convolutionless-type master equations with timedependent reaction rates [23]. Here, we adopt the latter-type non-Markovian master equation

$$
\begin{aligned}
\frac{\mathrm{d}}{\mathrm{d} t} P(n ; t) & =c_{0}(1+\psi(t)) v(t)[P(n-1 ; t)-P(n ; t)] \\
& +a_{1} v(t)[(n+1) P(n+1 ; t)-n P(n ; t)], \\
\psi(t) & =\sum_{j} \frac{\kappa_{0 j}}{\left[1+\kappa_{1 j}\left(t-T_{j}\right)^{2}\right]^{\alpha_{j}}}, \\
v(t) & =\frac{v_{0}}{1+v_{1} t}
\end{aligned}
$$

where $\psi(t)$ is the superposition of the Lorentzian-type functions including sets of real parameters $\left\{\left(\kappa_{0 j}, \kappa_{1 j}, T_{j}, \alpha_{j}\right)\right\}_{j}$ and $v(t)$ is a monotonically increasing function with two real parameters $\left(v_{0}, v_{1}\right)$. The time evolution of the PDF is exactly given by

$$
\begin{aligned}
P(n ; t) & =\frac{[\Phi(t)]^{n}}{n !} \exp [-\Phi(t)], \\
\Phi(t) & =\frac{c_{0}}{a_{1}}\left[1-\frac{1}{\left(1+v_{1} t\right)^{\frac{a_{1} v_{0}}{v_{1}}}}\right]+\frac{c_{0} \theta(t)}{\left(1+v_{1} t\right)^{\frac{a_{1} v_{0}}{v_{1}}}}, \\
\theta(t) & =\int_{0}^{t} v_{0}\left(1+v_{1} t^{\prime}\right)^{\frac{a_{1} v_{0}}{v_{1}}-1} \psi\left(t^{\prime}\right) \mathrm{d} t^{\prime} .
\end{aligned}
$$

The corresponding PDFs of the interarrival times of defects and the lifetimes of holes and MAWs are readily obtained as

$$
f_{n M}(t)=-\frac{\mathrm{d} \Phi(t)}{\mathrm{d} t} \exp [-\Phi(t)]
$$

Since $\Phi(t)$ satisfies $\lim _{t \rightarrow \infty} \Phi(t)=c_{0} / a_{1}, P(n ; t)$ leads to the Poisson distribution at stationary state, which is consistent with the result of the number statistics in the previous section. In a spe- 
cial case, $\psi(t)=0$ for MAWs, $f_{n M}(t)$ is simplified as

$$
f_{n M}(t)=\frac{c_{0} v_{0}}{v_{1}}\left(1+v_{1} t\right)^{-\frac{a_{1} v_{0}}{v_{1}}-1} \exp \left[-\frac{c_{0}}{a_{1}}\left(1-\left(1+v_{1} t\right)^{-\frac{a_{1} v_{0}}{v_{1}}}\right)\right]
$$

The theoretical PDFs of the interarrival times of defects and the lifetimes of holes and MAWs, $f_{n M}(t)$, are compared with those of the numerical results in Fig. 6. For the interarrival times of defects, $\tau_{D}, f_{n M}\left(\tau_{D}\right)$ captures the "power-law" behaviors for both low and high values of $\tau_{D}$ and the specific peaks involved in a period of the zigzag motions. The non-Markovian theoretical PDF $f_{n M}\left(l_{H}\right)$, for the lifetimes of holes, $l_{H}$, include a higher mode at $T_{1}=3.76$ in addition to the main peak at $T_{0}=0.48$, which may be generated from the nonlinearity of the system. The Markovian description, $f_{M}\left(l_{M}\right)$, and the non-Markovian one, $f_{n M}\left(l_{M}\right)$, are compared for the lifetimes of MAWs. The non-Markovian PDF $f_{n M}\left(l_{M}\right)$ agrees quite well with the numerical result because of the effect of the time-scaling function describing the "power-law" relaxations for both low and high values of $l_{M}$. However, the double-exponential decay, which is characteristic of the Markovian $\operatorname{PDF} f_{M}\left(l_{M}\right)$, cannot capture the "power-law" relaxations. From these observations, we conclude that our non-Markovian model can describe the effects of both the zigzag motions and the long-memory in the birthdeath process.

\section{Conclusions}

The 1D CGLE exhibits defect turbulence associated with the $\mathrm{BF}$ instability in large systems. From the point of view of the local structures, the defect turbulence can be successfully described by many-body interactions among defects, holes, and MAWs.

In this research, we have investigated defect turbulence via the birth-death process of the local structures. After identifying defects, holes and MAWs, we performed an appropriate statistical analysis for the number fluctuations, the interarrival times of defects, and the lifetimes of holes and MAWs. All the PDFs of the number of each local structure are subjected to the Poisson statistics, which is consistent with the analytical result derived from the master equation based on the local interaction rules among the local structures. In addition, we have shed light on the existence of long-memory and specific time scales in the interarrival times of defects and the lifetimes of holes and MAWs and have elucidated that their characteristics- "power laws" and specific peaks in the PDFs - can be identified by the non-Markovian master equation with time-dependent reaction rates. The non-Markovian master equation is characterized by the slowly varying time scale function and the superposition of the Lorentzian type functions, which are reflect the power-laws and the specific time peaks in the PDFs, respectively. These characteristics imply that the rather deterministic processes of the local structures, such as traveling holes and MAWs with long lifetimes and zigzag motions of holes, remain in the defect turbulence. In other words, the defect turbulence is not fully random spatiotemporal dynamics. The nature can be ascribed to the degree of freedom strictly limited in 1D systems. Actually, the PDF of lifetimes of defects in the 2D CGLE has a Markovian property, namely an exponential decay [24].

To the best of our knowledge, this is the first time that the statistical properties of local interactions involved in the appropriately distinguished local structures in defect turbulence of the 1D CGLE have been investigated. Although the parameters of the CGLE in Eq. (1) were fixed as $\left(c_{1}, c_{2}\right)=(1.5,-1.2)$, the same dynamical and statistical properties reported here can be observed in the parameter range of the defect turbulence (cf. the phase diagrams in Refs. [7, 10, 12]). On the other hand, the dynamical and statistical properties in the region of other spatiotemporal dynamics have not been elucidated involving the detailed local structures and constructing their interactions. Moreover, detailed identification of local structures in higher dimension has yet to be made. In $2 \mathrm{D}$ systems, the number statistics of topological defects have been thoroughly studied in the context of birth-death processes and revealed to be sub-Poisson statistics [19]. However, detailed classifications for local structures in 2D systems is not enough to construct local interaction rules because the topological defect may not be the only local structure. Therefore, further work is needed to clarify local structures in 2D systems and to uncover dynamical properties such as the existence of long-memory and/or characteristic time scales.

\section{Acknowledgements}

Y.U. is grateful for Grant-in-Aid for JSPS Fellows No. 25.374. H.K. is grateful for Grant-in-Aid for Challenging Exploratory Research No. 24650147.

\section{References}

[1] M. C. Cross, P. C. Hohenberg, Pattern formation outside of equilibrium, Rev. Mod. Phys. 65 (1993) 851-1112.

[2] I. S. Aranson, L. Kramer, The world of the complex Ginzburg-Landau equation, Rev. Mod. Phys. 74 (2002) 99-143.

[3] K. E. Daniels, C. Beck, E. Bodenschatz, Defect turbulence and generalized statistical mechanics, Physica D 193 (2004) 208-217.

[4] C. Beta, A. S. Mikhailov, H. H. Rotermund, G. Ertl, Defect-mediated turbulence in a catalytic surface reaction, Europhys. Lett. 75 (2006) 868874.

[5] F. X. Witkowski, L. J. Leon, P. A. Penkoske, W. R. Giles, M. L. Spano, W. L. Ditto, A. T. Winfree, Spatiotemporal evolution of ventricular fibrillation, Nature 392 (1998) 78-82.

[6] N. Bekki, Y. Harada, H. Kanai, Bekki-Nozaki hole in traveling excited waves on human cardiac interventricular septum, J. Phys. Soc. Jpn. 81 (2012) 073801.

[7] H. Chaté, Spatiotemporal intermittency regimes of the one-dimensional complex Ginzburg-Landau equation, Nonlinearity 7 (1994) 185-204.

[8] H. Chaté, P. Manneville, Phase diagram of the two-dimensional complex Ginzburg-Landau equation, Physica A 224 (1996) 348-368.

[9] W. van Saarloos, P. C. Hohenbergn, Fronts, pulses, sources and sinks in generalized complex Ginzburg-Landau equations, Physica D 56 (1992) 303-367.

[10] M. van Hecke, Building blocks of spatiotemporal intermittency, Phys. Rev. Lett. 80 (1998) 1896-1899.

[11] M. Howard, M. van Hecke, Hole-defect chaos in the one-dimensional complex Ginzburg-Landau equation, Phys. Rev. E 68 (2003) 026213.

[12] L. Brusch, M. G. Zimmermann, M. van Hecke, M. Bär, A. Torcini, Modulated amplitude waves and the transition from phase to defect chaos, Phys. Rev. Lett. 85 (2000) 86-89. 
[13] L. Brusch, A. Torcini, M. van Hecke, M. G. Zimmermann, M. Bär, Modulated amplitude waves and defect formation in the one-dimensional complex Ginzburg-Landau equation, Physica D 160 (2001) 127-148.

[14] M. van Hecke, Coherent and incoherent structures in systems described by the 1D CGLE: Experiments and identification, Physica D 174 (2003) 134-151.

[15] J. Sherratt, M. J. Smith, Transition to spatiotemporal chaos via stationary branching shocks and holes, Physica D 241 (2012) 1671-1679.

[16] J. B. Gonpe Tafo, L. Nana, T. C. Kofane, Time-delay autosynchronization of defect turbulence in the cubic-quintic complex Ginzburg-Landau equation, Phys. Rev. E 88 (2013) 032911.

[17] Y. Uciyama, H. Konno, Statistical characterization of hole turbulence in 1D complex Ginzburg-Landau equation, Proceedings of the 20th Australian Institute of Physics Congress, New South Wales (2012), No. 00303 (CD-ROM).

[18] Y. Uciyama, H. Konno, Statistical description of wave interactions in 1D defect turbulence, Int. J. Phys. Nat. Sci. Eng. 7 (2013) 20-25.

[19] L. Gil, J. Lega, J. L. Meunier, Statistical properties of defect-mediated turbulence, Phys. Rev. A 41 (1990) 1138-1141.

[20] M. Howard, M. van Hecke, Hole-defect chaos in the one-dimensional complex Ginzburg-Landau equation, Phys. Rev. E 68 (2003) 02613.

[21] C. Gardiner, Stochastic Methods: A Handbook for the Natural and Social Sciences, 4th edition, Springer, 2009.

[22] J. Klafter, I. M. Sokolov, First Steps in Random Walks: From Tools to Applications, Oxford University Press, 2011

[23] H. Konno, On the exact solution of a generalized Polya process, Adv. Math. Phys. 2010 (2010) 504267.

[24] C. Huepe, H. Riecke, Statistics of defect trajectories in spatio-temporal chaos in inclined layer convection and the complex Ginzburg-Landau equation, CHAOS 14 (2004) 864-874. 